\title{
BIG FIELDS THAT ARE NOT LARGE
}

\author{
BARRY MAZUR AND KARL RUBIN
}

(Communicated by Romyar T. Sharifi)

\begin{abstract}
A subfield $K$ of $\overline{\mathbb{Q}}$ is large if every smooth curve $C$ over $K$ with a $K$-rational point has infinitely many $K$-rational points. A subfield $K$ of $\overline{\mathbb{Q}}$ is big if for every positive integer $n, K$ contains a number field $F$ with $[F: \mathbb{Q}]$ divisible by $n$. The question of whether all big fields are large seems to have circulated for some time, although we have been unable to find its origin. In this paper we show that there are big fields that are not large.
\end{abstract}

\section{INTRODUCTION}

Definition 1.1. Following Pop [10, we say that a field $K$ is large if for every smooth curve $C$ defined over $K$, if the set of $K$-rational points $C(K)$ is non-empty then $C(K)$ is infinite. Equivalently (see [10, Proposition 1.1]), $K$ is large if for every irreducible variety $V$ defined over $K$ with a smooth $K$-rational point, $V(K)$ is Zariski dense in $V$.

Large fields (sometimes called ample fields) play a role in a number of basic conjectures regarding fields of algebraic numbers of infinite degree. For example, a conjecture of Shafarevich asserts that the absolute Galois group of $\mathbb{Q}^{\text {ab }}$, the maximal abelian extension of $\mathbb{Q}$, is isomorphic to the free profinite group with countably infinitely many generators. It follows from a theorem of Pop [10, Theorem 2.1] that if $\mathbb{Q}^{\mathrm{ab}}$ is large, then Shafarevich's conjecture holds. For more about large fields, see Section 5 below.

Recall that a supernatural number is a formal product

$$
\prod_{p} p^{n_{p}}
$$

where $p$ runs through all rational primes and $0 \leq n_{p} \leq \infty$, with the obvious notion of divisibility. If $K / L$ is an algebraic extension of fields, then we define the degree of $K$ over $L$

$$
[K: L]:=\operatorname{lcm}\{[F: L]: F \text { is a finite extension of } L \text { in } K\} .
$$

Definition 1.2. We say that a field $K \subset \overline{\mathbb{Q}}$ is $\operatorname{big}$ if $[K: \mathbb{Q}]=\prod_{p} p^{\infty}$. Equivalently, $K$ is big if for every positive integer $n, K$ contains a number field $F$ with $[F: \mathbb{Q}]$ divisible by $n$.

The main result of this note is the following.

Theorem 1.3. There are (uncountably many) big fields that are not large.

Received by the editors May 1, 2020, and, in revised form, May 18, 2020, June 11, 2020, and July 29, 2020.

2020 Mathematics Subject Classification. Primary 11R04, 11U05, 14 G05.

(C) 2020 by the authors under Creative Commons Attribution-Noncommercial 3.0 License (CC BY NC 3.0) 
More precisely, we will exhibit a non-empty set $\mathcal{S}_{0}$ of elliptic curves over $\mathbb{Q}$ such that for every $E \in \mathcal{S}_{0}$, there are uncountably many big fields $K$ such that $E(K)$ has exactly one rational point.

\section{2. $\mathcal{L}$-TOWERS}

Definition 2.1. Suppose $K$ is a number field, and $\mathcal{L}:=\left(\ell_{1}, \ell_{2}, \ldots\right)$ is a sequence of rational primes. The sequence $\mathcal{L}$ can be either infinite or finite, and the primes $\ell_{i}$ need not be distinct. We call an extension $K_{\infty} / K$ an $\mathcal{L}$-tower if there is a sequence of number fields $K=K_{0} \subset K_{1} \subset K_{2} \subset \cdots$ such that

(i) $K_{\infty}=\cup_{i} K_{i}$,

(ii) $K_{i} / K_{i-1}$ is cyclic of degree $\ell_{i}$,

(iii) for every $i>1$ there are primes $\mathfrak{p}_{i}, \mathfrak{p}_{i}^{\prime}$ of degree 1 of $K_{i}$, lying above the same prime of $K_{i-1}$, such that $\mathfrak{p}_{i}$ ramifies in $K_{i+1} / K_{i}$, but $\mathfrak{p}_{i}^{\prime}$ does not.

Note that if $K_{\infty} / K$ is an $\mathcal{L}$-tower, then $\left[K_{\infty}: K\right]=\prod_{i} \ell_{i}$.

Lemma 2.2. Suppose the chain of number fields $K_{i-1} \subset K_{i} \subset K_{i+1}$ is part of an $\mathcal{L}$-tower $K_{\infty} / K$, in the notation of Definition 2.1. Then there is no Galois extension $F / K_{i-1}$ such that $F K_{i}=K_{i+1}$.

Proof. Suppose $F / K_{i-1}$ is Galois. By Definition 2.1 there are primes $\mathfrak{p}, \mathfrak{p}^{\prime}$ of $K_{i}$, lying above the same prime $\mathfrak{q}$ of $K_{i-1}$, such that $\mathfrak{p}$ ramifies in $K_{i+1} / K_{i}$, but $\mathfrak{p}^{\prime}$ does not. Note that since $K_{i} / K_{i-1}$ is cyclic of prime degree and $\mathfrak{p} \neq \mathfrak{p}^{\prime}$, $\mathfrak{q}$ must split completely in $K_{i} / K_{i-1}$. Thus the diagram

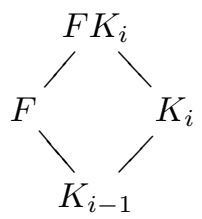

shows that

$\mathfrak{p}$ ramifies in $F K_{i} / K_{i} \Longleftrightarrow \mathfrak{q}$ ramifies in $F / K_{i-1} \Longleftrightarrow \mathfrak{p}^{\prime}$ ramifies in $F K_{i} / K_{i}$.

Thus $F K_{i} \neq K_{i+1}$, and the lemma follows.

Lemma 2.3. Suppose $\mathcal{L}$ is a sequence of rational primes, $K$ is a number field, and $K_{\infty}=\cup K_{i}$ is an $\mathcal{L}$-tower over $K$ as in Definition 2.1. Then:

(i) for every $i \geq 0$, the maximal abelian extension of $K_{i}$ in $K_{\infty}$ is $K_{i+1}$,

(ii) if $T$ is a Galois extension of $K$ (possibly of infinite degree) and $K_{1} \cap T=K$, then $K_{i} \cap T=K$ for every $i \geq 1$.

Proof. Suppose that there is an abelian extension $L$ of $K_{i}$ contained in $K_{\infty}$ and properly containing $K_{i+1}$. Without loss of generality we may assume that $\left[L: K_{i+1}\right]$ is prime. Choose $\alpha \in K_{\infty}$ such that $L=K_{i+1}(\alpha)$, and let $n$ be such that $\alpha \in K_{n+1}$ but $\alpha \notin K_{n}$. Since $L \supsetneq K_{i+1}$ we have $n>i$.

Since $\alpha \notin K_{n}$ and $\left[K_{n+1}: K_{n}\right]$ is prime, we have $K_{n+1}=K_{n}(\alpha)=L K_{n}$. This contradicts Lemma 2.2 applied with $i=n$ and $F=L K_{n-1}$. This contradiction proves (i). 
Now suppose $T / K$ is Galois with group $G$, and $K_{1} \cap T=K$. We will show by induction that $K_{i} \cap T=K$ for every $i$. Suppose $K_{i} \cap T=K$, and consider the diagram

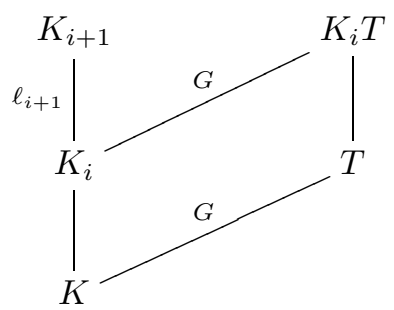

We either have $K_{i+1} \subset K_{i} T$ or $K_{i+1} \cap K_{i} T=K_{i}$. Suppose first that $K_{i+1} \subset K_{i} T$. Let $H:=\operatorname{Gal}\left(K_{i} T / K_{i+1}\right) \subset G$, so $T^{H} / K$ is abelian of degree $\ell_{i+1}$. Since $K_{i} \cap T=$ $K$, we have $K_{i+1}=T^{H} K_{i}$. This contradicts Lemma2.2 applied with $F=T^{H} K_{i-1}$, so we conclude that $K_{i+1} \cap K_{i} T=K_{i}$. Thus

$$
K_{i+1} \cap T=K_{i+1} \cap\left(K_{i} T \cap T\right)=\left(K_{i+1} \cap K_{i} T\right) \cap T=K_{i} \cap T=K .
$$

By induction this completes the proof of (ii).

Corollary 2.4. Suppose $K$ is a number field and $K_{\infty} / K$ is an algebraic extension. There is at most one sequence of rational primes $\mathcal{L}$ and one sequence of fields $\left(K_{0}, K_{1}, \ldots\right)$ that exhibits $K_{\infty} / K$ as an $\mathcal{L}$-tower.

Proof. Suppose $K_{\infty} / K$ is an $\mathcal{L}$-tower for some sequence $\mathcal{L}$. Applying Lemma 2.3(i) inductively shows that the sequence of fields $\left(K_{0}, K_{1}, \ldots\right)$ is uniquely determined, and then the fact that $\ell_{i}=\left[K_{i}: K_{i-1}\right]$ determines $\mathcal{L}$.

\section{SELmer GROUps}

If $F$ is a perfect field, $G_{F}$ will denote its absolute Galois group $\operatorname{Gal}(\bar{F} / F)$.

For this section fix a number field $K$, an elliptic curve $E$ defined over $K$, and a rational prime $\ell$ such that

$$
\text { the natural map } G_{K} \rightarrow \operatorname{Aut}(E[\ell]) \cong \mathrm{GL}_{2}\left(\mathbb{F}_{\ell}\right) \text { is surjective. }
$$

Let $L$ be either $K$ itself or a cyclic extension of $K$ of degree $\ell$.

For every place $v$ of $K$, let $K_{v}$ denote the completion of $K$ at $v$, let $L_{v}$ denote the completion of $L$ at some place above $v$, and let $\mathcal{H}_{\ell}\left(L_{v} / K_{v}\right)$ be the "local condition" subspace of $H^{1}\left(K_{v}, E[\ell]\right)$ defined in [6, Definition 7.1]. We will not repeat the definition here, but the following four propositions list all the properties of the subspaces $\mathcal{H}_{\ell}\left(L_{v} / K_{v}\right)$ that we need.

If $v$ is nonarchimedean with residue characteristic different from $\ell$, and $E$ has good reduction at $v$, let $K_{v}^{\text {ur }}$ denote the maximal unramified extension of $K_{v}$ and

$$
H_{\mathrm{ur}}^{1}\left(K_{v}, E[\ell]\right):=H^{1}\left(K_{v}^{\mathrm{ur}} / K_{v}, E[\ell]\right) \subset H^{1}\left(K_{v}, E[\ell]\right) .
$$

\section{Proposition 3.2.}

(i) $\operatorname{dim}_{\mathbb{F}_{\ell}} \mathcal{H}_{\ell}\left(L_{v} / K_{v}\right)=\frac{1}{2} \operatorname{dim}_{\mathbb{F}_{\ell}} H^{1}\left(K_{v}, E[\ell]\right)$.

(ii) If $L_{v}=K_{v}$ then $\mathcal{H}_{\ell}\left(L_{v} / K_{v}\right)$ is the image of the Kummer map $E\left(K_{v}\right) / \ell E\left(K_{v}\right) \rightarrow H^{1}\left(K_{v}, E[\ell]\right)$.

Proof. Assertion (i) holds because $\mathcal{H}_{\ell}\left(L_{v} / K_{v}\right)$ is a maximal isotropic subspace of $H^{1}\left(K_{v}, E[\ell]\right)$ for the local Tate pairing (see for example [4, Proposition 4.4]). Assertion (ii) is explained in [6, Definition 7.1]. 
Proposition 3.3. If $v \nmid \ell \infty, E$ has good reduction at $v$, and $L_{v} / K_{v}$ is ramified, then $\mathcal{H}_{\ell}\left(L_{v} / K_{v}\right) \cap H_{\mathrm{ur}}^{1}\left(K_{v}, E[\ell]\right)=0$.

Proof. This is [6, Proposition 7.8(ii)].

Proposition 3.4. If $v \nmid \ell \infty, L_{v} / K_{v}$ is unramified, $E$ has good reduction at $v$, and $\phi_{v} \in \operatorname{Gal}\left(K_{v}^{\mathrm{ur}} / K\right)$ is the Frobenius generator, then:

(i) $\mathcal{H}_{\ell}\left(L_{v} / K_{v}\right)=H_{\mathrm{ur}}^{1}\left(K_{v}, E[\ell]\right)$,

(ii) $\operatorname{dim}_{\mathbb{F}_{\ell}} \mathcal{H}_{\ell}\left(L_{v} / K_{v}\right)=\operatorname{dim}_{\mathbb{F}_{\ell}}\left(E[\ell]^{\phi_{v}=1}\right)$,

(iii) the map $H_{\mathrm{ur}}^{1}\left(K_{v}, E[\ell]\right) \rightarrow E[\ell] /\left(\phi_{v}-1\right) E[\ell]$ given by evaluating cocycles at $\phi_{v}$ is a well-defined isomorphism.

Proof. Assertions (i) and (iii) are [6, Lemma 7.3], and (ii) is [6, Lemma 7.2].

Let $\Delta_{E}$ denote the discriminant of some Weierstrass model of $E$.

Proposition 3.5. If $\ell=2, v \nmid 2 \infty, E$ has multiplicative reduction at $v, \operatorname{ord}_{v}\left(\Delta_{E}\right)$ is odd, and $L_{v} / K_{v}$ is unramified, then $\mathcal{H}_{\ell}\left(L_{v} / K_{v}\right)=\mathcal{H}_{\ell}\left(K_{v} / K_{v}\right)$.

Proof. This is [5, Lemma 2.10(iii)].

Definition 3.6. The relative Selmer group $\operatorname{Sel}(L / K, E[\ell])$ is the subgroup of $H^{1}(K, E[\ell])$ defined by

$$
\operatorname{Sel}(L / K, E[\ell]):=\left\{c \in H^{1}(K, E[\ell]): \operatorname{loc}_{v}(c) \in \mathcal{H}_{\ell}\left(L_{v} / K_{v}\right) \text { for every } v\right\}
$$

where $\operatorname{loc}_{v}: H^{1}(K, E[\ell]) \rightarrow H^{1}\left(K_{v}, E[\ell]\right)$ is the localization map at $v$.

When $L=K$, Proposition 3.2 (ii) shows that $\operatorname{Sel}(L / K, E[\ell])$ is the standard $\ell$-Selmer group of $E / K$, and we denote it by $\operatorname{Sel}(K, E[\ell])$.

When $\ell=[L: K]=2, \operatorname{Sel}(L / K, E[\ell])$ is the standard 2-Selmer group of the quadratic twist $E^{L} / K$ (see [6, Lemma 8.4]).

These relative Selmer groups are useful to us because of the following proposition.

\section{Proposition 3.7.}

(i) If $\operatorname{Sel}(L / K, E[\ell])=0$ then $\operatorname{rank} E(L)=\operatorname{rank} E(K)$.

(ii) If $\operatorname{rank} E(L)=\operatorname{rank} E(K)$ and $L / K$ is ramified at two primes of good reduction for $E$ with different residue characteristics, then $E(L)=E(K)$.

Proof. By [6, Proposition 8.8],

$\operatorname{rank} E(K) \leq \operatorname{rank} E(L) \leq \operatorname{rank} E(K)+(\ell-1) \operatorname{dim}_{\mathbb{F}_{\ell}} \operatorname{Sel}(L / K, E[\ell])$.

Assertion (i) follows directly.

Suppose now that $\operatorname{rank} E(L)=\operatorname{rank} E(K)$ but $E(L)$ properly contains $E(K)$. Then we can fix a point $x \in E(L)$ such that $x \notin E(K)$ but $p x \in E(K)$ for some rational prime $p$. Since $x \notin E(K)$ and $[L: K]$ is prime, we must have $K(x)=L$. But $K(x) / K$ can ramify only at places of bad reduction and primes above $p$ [13. Theorem VII.7.1], so this contradicts our assumption that $L / K$ ramifies at good primes with two distinct residue characteristics.

Lemma 3.8. Suppose that $c$ is a cocycle representing a nonzero class in $H^{1}(K, E[\ell])$. Let $F=K(E[\ell])$. The restriction of $c$ to $G_{F}$ induces a surjective homomorphism

$$
G_{F} \longrightarrow E[\ell] \text {. }
$$


Proof. Using (3.1), the kernel of restriction $H^{1}(K, E[\ell]) \rightarrow H^{1}(F, E[\ell])$ is $H^{1}(F / K, E[\ell]) \cong H^{1}\left(\mathrm{GL}_{2}\left(\mathbb{F}_{\ell}\right), \mathbb{F}_{\ell}^{2}\right)=0$. Therefore the restriction of $c$ to $G_{F}$ is a nonzero homomorphism $\tilde{c}: G_{F} \rightarrow E[\ell]$. Since $\tilde{c}$ is the restriction of a class defined over $K$, we have that $\tilde{c}$ is $G_{K}$-equivariant, and in particular the image of $\tilde{c}$ is stable under $G_{K}$. By (3.1) it follows that $\tilde{c}$ is surjective.

Definition 3.9. If $\mathfrak{a}$ is an ideal of $\mathcal{O}_{K}$, define relaxed-at- $\mathfrak{a}$ and strict-at-a Selmer groups, respectively, by

$$
\begin{aligned}
& \operatorname{Sel}(K, E[\ell])^{\mathfrak{a}}:=\left\{c \in H^{1}(K, E[\ell]): \operatorname{loc}_{v}(c) \in \mathcal{H}_{\ell}\left(K_{v} / K_{v}\right) \text { for every } v \nmid \mathfrak{a}\right\}, \\
& \operatorname{Sel}(K, E[\ell])_{\mathfrak{a}}:=\left\{c \in \operatorname{Sel}(K, E[\ell]): \operatorname{loc}_{v}(c)=0 \text { for every } v \mid \mathfrak{a}\right\} .
\end{aligned}
$$

Note that

$$
\operatorname{Sel}(K, E[\ell])_{\mathfrak{a}} \subset \operatorname{Sel}(K, E[\ell]) \subset \operatorname{Sel}(K, E[\ell])^{\mathfrak{a}} .
$$

If $\Sigma$ is a finite set of places of $K$ containing all archimedean places, then the ring of $\Sigma$-integers of $K$ is

$$
\mathcal{O}_{K, \Sigma}:=\left\{x \in K: x \in \mathcal{O}_{K_{v}} \text { for every } v \notin \Sigma\right\} .
$$

Definition 3.10. From now on let $\Sigma$ be a finite set of places of $K$ containing all places where $E$ has bad reduction, all places dividing $\ell \infty$, and large enough so that

- the primes in $\Sigma$ generate the ideal class group of $K$,

- the natural map $\mathcal{O}_{K, \Sigma}^{\times} /\left(\mathcal{O}_{K, \Sigma}^{\times}\right)^{\ell} \rightarrow \prod_{v \in \Sigma} K_{v}^{\times} /\left(K_{v}^{\times}\right)^{\ell}$ is injective

(this is possible by [1, Lemma 6.1]). Define a set $\mathcal{P}$ of primes of $K$ by

$$
\mathcal{P}:=\{\mathfrak{p} \notin \Sigma: \mathbf{N p} \equiv 1 \quad(\bmod \ell)\}
$$

and define a partition of $\mathcal{P}$ into disjoint subsets $\mathcal{P}_{i}$ for $0 \leq i \leq 2$ by

$$
\mathcal{P}_{i}:=\left\{\mathfrak{p} \in \mathcal{P}: \operatorname{dim}_{\mathbb{F}_{\ell}} H_{\mathrm{ur}}^{1}\left(K_{\mathfrak{p}}, E[\ell]\right)=i\right\} .
$$

(Equivalently by Proposition 3.4. $\left.\mathcal{P}_{i}:=\left\{\mathfrak{p} \in \mathcal{P}: \operatorname{dim}_{\mathbb{F}_{\ell}} E\left(K_{\mathfrak{p}}\right)[\ell]\right)=i\right\}$.) If $\mathfrak{a}$ is an ideal of $\mathcal{O}_{K}$, let $\mathcal{P}_{1}(\mathfrak{a})$ be the set of all $\mathfrak{p} \in \mathcal{P}_{1}$ such that the localization map

$$
\operatorname{Sel}(K, E[\ell])_{\mathfrak{a}} \stackrel{\operatorname{loc}_{\mathfrak{p}}}{\longrightarrow} H_{\mathrm{ur}}^{1}\left(K_{\mathfrak{p}}, E[\ell]\right)
$$

is nonzero.

The next proposition is a modification of [6, Proposition 9.10].

\section{Proposition 3.11.}

(i) The sets $\mathcal{P}_{0}$ and $\mathcal{P}_{1}$ have positive density.

(ii) Suppose $\mathfrak{a}$ is an ideal of $\mathcal{O}_{K}$ such that $\operatorname{Sel}(K, E[\ell])_{\mathfrak{a}}$ is nonzero. Then $\mathcal{P}_{1}(\mathfrak{a})$ has positive density, and if $\mathfrak{p} \in \mathcal{P}_{1}(\mathfrak{a})$ then

$$
\operatorname{dim}_{\mathbb{F}_{\ell}} \operatorname{Sel}(K, E[\ell])_{\mathfrak{a p}}=\operatorname{dim}_{\mathbb{F}_{\ell}} \operatorname{Sel}(K, E[\ell])_{\mathfrak{a}}-1 .
$$

Proof. Let $F:=K(E[\ell])$, and fix $i=0$ or 1. It follows from the surjection (3.1) that $\operatorname{Gal}\left(F / K\left(\boldsymbol{\mu}_{\ell}\right)\right) \cong \mathrm{SL}_{2}\left(\mathbb{F}_{\ell}\right)$. Fix $\tau_{i} \in G_{K\left(\boldsymbol{\mu}_{\ell}\right)}$ such that $\operatorname{dim}_{\mathbb{F}_{\ell}} E[\ell]^{\tau_{i}=1}=i$.

Suppose $\mathfrak{p}$ is a prime of $K$ whose Frobenius conjugacy class in $\operatorname{Gal}(K(E[\ell]) / K)$ is the class of $\tau_{i}$. Since $\tau_{i}$ fixes $\boldsymbol{\mu}_{\ell}$, we have that $\boldsymbol{\mu}_{\ell} \subset K_{\mathfrak{p}}^{\times}$so $\mathbf{N p} \equiv 1(\bmod \ell)$ and therefore by definition $\mathfrak{p} \in \mathcal{P}$. By Proposition 3.4 we have

$$
\operatorname{dim}_{\mathbb{F}_{\ell}} \mathcal{H}_{\ell}\left(L_{v} / K_{v}\right)=\operatorname{dim}_{\mathbb{F}_{\ell}} H_{\mathrm{ur}}^{1}\left(K_{v}, E[\ell]\right)=\operatorname{dim} E[\ell]^{\tau_{i}=1}=i,
$$

so $\mathfrak{p} \in \mathcal{P}_{i}$. It follows from the Cebotarev Theorem that $\mathcal{P}_{i}$ has positive density. This is (i). 
Fix an ideal $\mathfrak{a}$ of $\mathcal{O}_{K}$ and suppose that $c$ is a cocycle representing a nonzero element of $\operatorname{Sel}(K, E[\ell])_{\mathfrak{a}}$. Let $\tau_{1}$ be as above. By Lemma 3.8, the restriction of $c$ to $G_{F}$ induces a surjective (and therefore nonzero) homomorphism

$$
\tilde{c}: G_{F} \longrightarrow E[\ell] /\left(\tau_{1}-1\right) E[\ell] .
$$

Since $\tilde{c} \neq 0$, we can find $\gamma \in G_{F}$ such that $\tilde{c}(\gamma) \neq-c\left(\tau_{1}\right)$ in $E[\ell] /\left(\tau_{1}-1\right) E[\ell]$. Then

$$
c\left(\gamma \tau_{1}\right) \notin\left(\tau_{1}-1\right) E[\ell]=\left(\gamma \tau_{1}-1\right) E[\ell] .
$$

Let $N$ be a Galois extension of $K$ containing $F$ and such that the restriction of $c$ to $G_{F}$ factors through $\operatorname{Gal}(N / F)$. If $\mathfrak{p}$ is a prime whose Frobenius conjugacy class in $\operatorname{Gal}(N / K)$ is the class of $\gamma \tau_{1}$, then by Proposition 3.4(iii) we have $\operatorname{loc}_{\mathfrak{p}}(c) \neq 0$, so $\mathfrak{p} \in \mathcal{P}_{1}(\mathfrak{a})$. Now the Cebotarev Theorem shows that $\mathcal{P}_{1}(\mathfrak{a})$ has positive density.

If $\mathfrak{p} \in \mathcal{P}_{1}(\mathfrak{a})$ then we have an exact sequence

$$
0 \longrightarrow \operatorname{Sel}(K, E[\ell])_{\mathfrak{a p}} \longrightarrow \operatorname{Sel}(K, E[\ell])_{\mathfrak{a}} \stackrel{\operatorname{loc}_{\mathfrak{p}}}{\longrightarrow} H_{\mathrm{ur}}^{1}\left(K_{\mathfrak{p}}, E[\ell]\right) \longrightarrow 0
$$

where the right-hand map is surjective because it is nonzero and the target space is one-dimensional. This completes the proof of (ii).

Definition 3.12. Suppose $T$ is a finite set of primes of $K$, disjoint from $\Sigma$. If $\ell=2$ let $\Sigma_{0}$ denote the subset of $\Sigma$ consisting of all primes $\mathfrak{p}$ where $E$ has multiplicative reduction and such that $\operatorname{ord}_{\mathfrak{p}}\left(\Delta_{E}\right)$ is odd. If $\ell>2$ let $\Sigma_{0}$ be the empty set. We say that an extension $L / K$ is $T$-ramified and $\Sigma$-split if

- every $\mathfrak{p} \in T$ is ramified in $L / K$, every $\mathfrak{p} \notin T$ is unramified in $L / K$,

- every $v \in \Sigma-\Sigma_{0}$ splits in $L / K$.

The next proposition is a modification of [6, Proposition 9.17].

Proposition 3.13. Let $r:=\operatorname{dim}_{\mathbb{F}_{\ell}} \operatorname{Sel}(K, E[\ell])$ and suppose $t \leq r$.

(i) There is a set of primes $T \subset \mathcal{P}_{1}$ of cardinality $t$ such that

$$
\operatorname{dim}_{\mathbb{F}_{\ell}} \operatorname{Sel}(K, E[\ell])_{\mathfrak{a}}=r-t,
$$

where $\mathfrak{a}:=\prod_{\mathfrak{p} \in T} \mathfrak{p}$.

(ii) If $T$ satisfies ( $i), T_{0}$ is a finite subset of $\mathcal{P}_{0}$, and $L / K$ is a cyclic extension of $K$ of degree $\ell$ that is $\left(T \cup T_{0}\right)$-ramified and $\Sigma$-split, then

$$
\operatorname{dim}_{\mathbb{F}_{\ell}} \operatorname{Sel}(L / K, E[\ell])=r-t .
$$

Proof. We will prove (i) by induction on $t$. If $t=0$, then $T$ is the empty set.

Suppose $T$ satisfies (i) for $t$, and $t<r$. Let $\mathfrak{a}:=\prod_{\mathfrak{p} \in T} \mathfrak{p}$. By Proposition 3.11(ii) we can find $\mathfrak{p} \in \mathcal{P}_{1}(\mathfrak{a})$ so that

$$
\operatorname{dim}_{\mathbb{F}_{\ell}} \operatorname{Sel}(K, E[\ell])_{\mathfrak{a p}}=r-t-1 .
$$

Then $T \cup\{\mathfrak{p}\}$ satisfies (i) for $t+1$. This proves (i).

Now suppose that $T$ satisfies (i), and let $\mathfrak{a}:=\prod_{\mathfrak{p} \in T} \mathfrak{p}$. Consider the exact sequences

$$
\begin{aligned}
0 \longrightarrow \operatorname{Sel}(K, E[\ell]) \longrightarrow \operatorname{Sel}(K, E[\ell])^{\mathfrak{a}} \stackrel{\oplus \operatorname{loc}_{\mathfrak{p}}}{\longrightarrow} \bigoplus_{\mathfrak{p} \in T} \frac{H^{1}\left(K_{\mathfrak{p}}, E[\ell]\right)}{H_{\mathrm{ur}}^{1}\left(K_{\mathfrak{p}}, E[\ell]\right)} \\
0 \rightarrow \operatorname{Sel}(K, E[\ell])_{\mathfrak{a}} \longrightarrow \operatorname{Sel}(K, E[\ell]) \stackrel{\oplus \operatorname{loc}_{\mathfrak{p}}}{\longrightarrow} \bigoplus_{\mathfrak{p} \in T} H_{\mathrm{ur}}^{1}\left(K_{\mathfrak{p}}, E[\ell]\right) .
\end{aligned}
$$


By global duality (see for example [3, Theorem 2.3.4]), the images of the two righthand maps in (3.14) are orthogonal complements of each other under the sum of the local Tate pairings. By our choice of $T$ the lower right-hand map is surjective, so the upper right-hand map is zero, i.e.,

$$
\left(\oplus_{\mathfrak{p} \in T} \operatorname{loc}_{\mathfrak{p}}\right)\left(\operatorname{Sel}(K, E[\ell])^{\mathfrak{a}}\right) \subset \bigoplus_{\mathfrak{p} \in T} H_{\mathrm{ur}}^{1}\left(K_{\mathfrak{p}}, E[\ell]\right) .
$$

Let $T_{0}$ be a finite subset of $\mathcal{P}_{0}$, let $\mathfrak{b}:=\prod_{\mathfrak{p} \in T_{0}} \mathfrak{p}$, and suppose $L$ is a cyclic extension of $K$ of degree $\ell$ that is $\left(T \cup T_{0}\right)$-ramified and $\Sigma$-split. By Propositions [3.4(i) and 3.5. we have $\mathcal{H}_{\ell}\left(L_{v} / K_{v}\right)=\mathcal{H}_{\ell}\left(K_{v} / K_{v}\right)$ if $v \notin T \cup T_{0}$. Thus by Definition 3.6. $\operatorname{Sel}(L / K, E[\ell])$ is the kernel of the map

$$
\operatorname{Sel}(K, E[\ell])^{\mathfrak{a} \mathfrak{b}} \stackrel{\oplus_{\mathfrak{p} \in T \cup T_{0}} \operatorname{loc}_{\mathfrak{p}}}{\longrightarrow} \bigoplus_{\mathfrak{p} \in T \cup T_{0}} H^{1}\left(K_{\mathfrak{p}}, E[\ell]\right) / \mathcal{H}_{\ell}\left(L_{\mathfrak{p}} / K_{\mathfrak{p}}\right) .
$$

We have $\mathcal{H}_{\ell}\left(L_{\mathfrak{p}} / K_{\mathfrak{p}}\right)=H^{1}\left(K_{\mathfrak{p}}, E[\ell]\right)=0$ for every $\mathfrak{p} \in \mathcal{P}_{0}$ by Propositions $3.2(\mathrm{i})$ and 3.4 (ii) and the definition of $\mathcal{P}_{0}$, so in fact $\operatorname{Sel}(L / K, E[\ell])$ is the kernel of the map

$$
\operatorname{Sel}(K, E[\ell])^{\mathfrak{a}} \stackrel{\oplus_{\mathfrak{p} \in T} \operatorname{loc}_{\mathfrak{p}}}{\longrightarrow} \bigoplus_{\mathfrak{p} \in T} H^{1}\left(K_{\mathfrak{p}}, E[\ell]\right) / \mathcal{H}_{\ell}\left(L_{\mathfrak{p}} / K_{\mathfrak{p}}\right) .
$$

By Proposition 3.3, $H_{\text {ur }}^{1}\left(K_{\mathfrak{p}}, E[\ell]\right) \cap \mathcal{H}_{\ell}\left(L_{\mathfrak{p}} / K_{\mathfrak{p}}\right)=0$ for every $\mathfrak{p} \in T$. Combining (3.15) and (3.16) shows that $\operatorname{Sel}(L / K, E[\ell])=\operatorname{Sel}(L / K, E[\ell])_{\mathfrak{a}}$, so by our choice of $T$ we have $\operatorname{dim}_{\mathbb{F}_{\ell}} \operatorname{Sel}(L / K, E[\ell])=r-t$. This proves (ii).

Proposition 3.17. Suppose $T$ is a finite subset of $\mathcal{P}_{0} \cup \mathcal{P}_{1}$. If $\ell=2$ suppose further that $E$ has a prime $\mathfrak{q} \nmid 2$ of multiplicative reduction such that $\operatorname{ord}_{\mathfrak{q}}\left(\Delta_{E}\right)$ is odd. Then there are a finite subset $T_{0} \subset \mathcal{P}_{0}$ and a cyclic extension $L / K$ of degree $\ell$ such that

(i) $L / K$ is $\left(T \cup T_{0}\right)$-ramified and $\Sigma$-split,

(ii) $K$ has a prime $\mathfrak{p}$ of degree 1 , unramified over $\mathbb{Q}$, such that $L / K$ is ramified at $\mathfrak{p}$ and unramified at all primes $\mathfrak{p}^{\prime} \neq \mathfrak{p}$ with the same residue characteristic as $\mathfrak{p}$.

Proof. Fix a prime $\mathfrak{p} \in \mathcal{P}_{0}$ of degree 1 , unramified over $\mathbb{Q}$, whose residue characteristic $p$ is different from the residue characteristics of all primes in $T$ (this is possible by Proposition 3.11(i)). Let $\Sigma_{p}:=\Sigma \cup\{v$ of $K: v \mid p\}$.

Define a set of global Galois characters

$$
\begin{aligned}
\mathcal{C}(T):=\left\{\chi \in \operatorname{Hom}\left(G_{K}, \boldsymbol{\mu}_{\ell}\right): \chi\right. & \text { is ramified at all } \mathfrak{p} \in T \text { and } \\
& \text { unramified at all primes not in } \left.\Sigma_{p} \cup T \cup \mathcal{P}_{0}\right\},
\end{aligned}
$$

and a set of tuples of local characters

$$
\Omega_{T}:=\prod_{v \in \Sigma_{p}} \operatorname{Hom}\left(G_{K_{v}}, \boldsymbol{\mu}_{\ell}\right) \times \prod_{v \in T} \operatorname{Hom}_{\mathrm{ram}}\left(G_{K_{v}}, \boldsymbol{\mu}_{\ell}\right)
$$

where $\operatorname{Hom}_{\text {ram }}\left(G_{K_{v}}, \boldsymbol{\mu}_{\ell}\right) \subset \operatorname{Hom}\left(G_{K_{v}}, \boldsymbol{\mu}_{\ell}\right)$ denotes the subset of ramified characters.

Suppose first that $\ell \geq 3$. Restriction gives a natural map of sets $\mathcal{C}(T) \rightarrow \Omega_{T}$, and by [2, Proposition 10.7] this map is surjective. Now take an element $\omega \in \Omega_{T}$ whose $v$-component $\omega_{v}$ is the trivial character if $v \in \Sigma_{p}$ is different from $\mathfrak{p}$, and 
such that $\omega_{\mathfrak{p}}$ is ramified. Let $\chi \in \mathcal{C}(T)$ be any character that restricts to $\omega$, and let $T_{0}$ be the set of primes (necessarily in $\mathcal{P}_{0}$ ) not in $T$ where $\chi$ is ramified. If $L$ is the cyclic extension of $K$ corresponding to $\chi$, then $L / K$ is $\left(T \cup T_{0}\right)$-ramified and $\Sigma$-split, and $L$ ramifies at $\mathfrak{p}$ but not at any other prime above $p$. This proves the proposition when $\ell \geq 3$.

When $\ell=2$ the proof is similar, except that the map $\eta: \mathcal{C}(T) \rightarrow \Omega_{T}$ is not surjective. However in this case, [2, Proposition 10.7] shows that the image of $\eta$ contains either our chosen $\omega \in \Omega_{T}$, or else $\omega^{\prime}$, where $\omega_{v}^{\prime}$ is $\omega_{v}$ if $v \neq \mathfrak{q}$, and $\omega_{\mathfrak{q}}^{\prime}$ is the nontrivial unramified quadratic character (where $\mathfrak{q}$ is the given prime of multiplicative reduction). The proof now proceeds exactly as in the case of odd $\ell$, using a character $\chi \in \mathcal{C}(T)$ that maps to either $\omega$ or $\omega^{\prime}$.

\section{Proof of Theorem 1.3}

Definition 4.1. Let $\mathcal{S}$ be the set of all elliptic curves $E$ over $\mathbb{Q}$ satisfying all of the following properties:

- for every prime $\ell$, the map $G_{\mathbb{Q}} \rightarrow \operatorname{Aut}(E[\ell]) \cong \mathrm{GL}_{2}\left(\mathbb{F}_{\ell}\right)$ is surjective,

- $E$ has discriminant $\Delta_{E} \equiv 1(\bmod 4)$,

- $E$ has a prime $q \nmid 2$ of multiplicative reduction such that $\operatorname{ord}_{q}\left(\Delta_{E}\right)$ is odd. Let $\mathcal{S}_{0}=\{E \in \mathcal{S}: E(\mathbb{Q})=0\}$.

Lemma 4.2. The set $\mathcal{S}_{0}$ is nonempty.

Proof. Let $E$ be the elliptic curve labelled 67.a1 in the $L$-functions and Modular Forms Database [9]. We will show that $E \in \mathcal{S}_{0}$.

The surjectivity of the map $G_{\mathbb{Q}} \rightarrow \operatorname{Aut}(E[\ell]) \cong \mathrm{GL}_{2}\left(\mathbb{F}_{\ell}\right)$ for every $\ell$ is stated in [9, curve 67.a1]; alternatively this follows directly from [11, Proposition 21] and the fact that $E$ has no rational isogenies [9, curve 67.a1]. The curve $E$ has multiplicative reduction at 67 , and its discriminant -67 is congruent to $1(\bmod 4)$. Thus $E \in \mathcal{S}$, and $E(\mathbb{Q})=0$ so $E \in \mathcal{S}_{0}$.

Remark 4.3. The elliptic curve $67 . a 1$ in the proof of Lemma4.2 has only one rational point. If we instead take $E$ to be the curve $37 . a 1$, then $E(\mathbb{Q}) \cong \mathbb{Z}$ and a similar argument shows that $E \in \mathcal{S}$.

For $E \in \mathcal{S}$, define $T_{E}$ to be the compositum of all the fields $\mathbb{Q}(E[\ell])$ for all primes $\ell$. Note that if $K$ is a number field and $K \cap T_{E}=\mathbb{Q}$, then for every prime $\ell$, the $\operatorname{map} G_{K} \rightarrow \operatorname{Aut}(E[\ell]) \cong \mathrm{GL}_{2}\left(\mathbb{F}_{\ell}\right)$ is surjective.

Proposition 4.4. Suppose $E \in \mathcal{S}$, and $K$ is a finite nontrivial extension of $\mathbb{Q}$ such that $K \cap T_{E}=\mathbb{Q}$ and $E(K)=E(\mathbb{Q})$. Then for each rational prime $\ell$ there are infinitely many cyclic extensions $L / K$ of degree $\ell$ such that:

(i) $E(L)=E(K)$,

(ii) $K$ has a prime $\mathfrak{p}$ of degree 1 , unramified over $\mathbb{Q}$, such that $L / K$ is ramified at $\mathfrak{p}$ and unramified at all primes $\mathfrak{p}^{\prime} \neq \mathfrak{p}$ with the same residue characteristic as $\mathfrak{p}$,

(iii) $L / K$ is ramified at (at least) two primes where $E$ has good reduction and that have different residue characteristics,

(iv) every place of $K$ dividing $\ell \infty$ splits completely in $L / K$.

Proof. Let $T$ be a finite subset of $\mathcal{P}_{1}$ satisfying Proposition 3.13(i) with $t:=$ $\operatorname{dim}_{\mathbb{F}_{\ell}} \operatorname{Sel}(K, E[\ell])$. Let $T^{\prime}$ be a finite subset of $T \cup \mathcal{P}_{0}$ containing $T$ and at least 
two primes in $\mathcal{P}_{0}$ with different residue characteristics. Now apply Proposition 3.17. with the set $T^{\prime}$ in place of $T$, to produce a cyclic extension $L / K$ that is $\left(T^{\prime} \cup T_{0}\right)$-ramified and $\Sigma$-split for some $T_{0} \subset \mathcal{P}_{0}$.

By Proposition 3.13 (ii) we have $\operatorname{Sel}(L / K, E[\ell])=0$, and then Proposition 3.7 shows that $E(L)=E(K)$. Assertion (ii) is Proposition 3.17(ii), and (iii) and (iv) follow from our choice of $T^{\prime}$ and Definition 3.12 of " $\Sigma$-split", since all places dividing $\ell \infty$ are in $\Sigma$.

Thus $L$ has the desired properties. By varying the set $T^{\prime}$ we can produce infinitely many such $L$.

Lemma 4.5. Suppose $E \in \mathcal{S}$. Then there is a real quadratic field $F$, ramified at 2 , such that $E(F)=E(\mathbb{Q})$ and $F \cap T_{E}=\mathbb{Q}$.

Proof. If $A$ is an elliptic curve over $\mathbb{Q}$ and $d \in \mathbb{Q}^{\times}$, let $A^{(d)}$ denote the quadratic twist of $A$ corresponding to $\mathbb{Q}(\sqrt{d}) / \mathbb{Q}$. Then $\left(A^{(d)}\right)^{\left(d^{\prime}\right)}=A^{\left(d d^{\prime}\right)}$ and

$$
\operatorname{rank} A(\mathbb{Q}(\sqrt{d}))=\operatorname{rank} A(\mathbb{Q})+\operatorname{rank} A^{(d)}(\mathbb{Q}) .
$$

Fix an elliptic curve $E \in \mathcal{S}$. Apply Proposition 4.4 to the curve $E^{(2)}$ with $K=\mathbb{Q}$ and $\ell=2$ to get a quadratic extension $L / \mathbb{Q}$ satisfying (i) through (iv) of Proposition 4.4. Write $L=\mathbb{Q}(\sqrt{D})$ with a squarefree integer $D$, and put $F:=\mathbb{Q}(\sqrt{2 D})$.

Applying (4.6) with $A=E^{(2)}$ and $d=D$ yields

$$
\operatorname{rank} E^{(2)}(L)=\operatorname{rank} E^{(2)}(\mathbb{Q})+\operatorname{rank} E^{(2 D)}(\mathbb{Q}) .
$$

By Proposition 4.4 (i) we have $E^{(2)}(L)=E^{(2)}(\mathbb{Q})$, so rank $E^{(2 D)}(\mathbb{Q})=0$. Applying (4.6) with $A=E$ and $d=2 D$ yields

$$
\operatorname{rank} E(F)=\operatorname{rank} E(\mathbb{Q})+\operatorname{rank} E^{(2 D)}(\mathbb{Q})
$$

so $\operatorname{rank} E(F)=\operatorname{rank} E(\mathbb{Q})$. By Proposition 4.4(iii,iv) $D$ is positive, odd, and divisible by at least 2 primes where $E$ has good reduction. Thus $F$ is a real field, ramified at 2, and $E(F)=E(\mathbb{Q})$ by Proposition 3.7(ii).

Since the discriminant $\Delta_{E} \equiv 1(\bmod 4)$, the quadratic field $\mathbb{Q}\left(\sqrt{\Delta_{E}}\right) \subset \mathbb{Q}(E[2])$ is unramified at 2 , so 2 is tamely ramified in $\mathbb{Q}(E[2]) / \mathbb{Q}$. Since $\Delta_{E}$ is odd, $E$ has good reduction at 2 , so 2 is unramified in $\mathbb{Q}(E[\ell]) / \mathbb{Q}$ for all odd primes $\ell$ [13, Theorem VII.7.1]. Hence 2 is tamely ramified in $T_{E} / \mathbb{Q}$, but 2 is wildly ramified in $F / \mathbb{Q}$, so $F \cap T_{E}=\mathbb{Q}$.

Theorem 4.7. Suppose $E \in \mathcal{S}$. Then for every infinite sequence $\mathcal{L}$ of rational primes with $\ell_{1}=2$, there are uncountably many totally real $\mathcal{L}$-towers $K_{\infty} / \mathbb{Q}$ such that $E\left(K_{\infty}\right)=E(\mathbb{Q})$.

Proof. We build an $\mathcal{L}$-tower inductively as follows. Let $K_{0}=\mathbb{Q}$ and let $K_{1}$ be a field $F$ as in Lemma 4.5. Now for each $i \geq 2$ apply Proposition 4.4 to produce a cyclic extension $K_{i} / K_{i-1}$ of degree $\ell_{i}$ such that $E\left(K_{i}\right)=E\left(K_{i-1}\right)=E(\mathbb{Q})$.

Since $K_{1} \cap T_{E}=F \cap T_{E}=\mathbb{Q}$, Lemma 2.3(ii) shows that $K_{i} \cap T_{E}=\mathbb{Q}$ and we can continue by induction. The result is an $\mathcal{L}$-tower $K_{\infty} / \mathbb{Q}$ such that $E\left(K_{\infty}\right)=E(\mathbb{Q})$. Since at each step Proposition 4.4 provides us with infinitely many possible $K_{i}$, and different choices give rise to distinct $\mathcal{L}$-towers by Corollary 2.4 , we obtain uncountably many $\mathcal{L}$-towers in this way.

Proof of Theorem 1.3. Let $\mathcal{L}$ be a sequence of rational primes such that

- $\ell_{1}=2$, and

- every prime occurs infinitely many times in $\mathcal{L}$. 
If $K_{\infty} / \mathbb{Q}$ is an $\mathcal{L}$-tower, then $K_{\infty}$ is a big field. We can apply Theorem 4.7 taking for $E \in \mathcal{S}_{0}$ the curve 67.a1 from the proof of Lemma 4.2. to produce uncountably many $\mathcal{L}$-towers $K_{\infty} / \mathbb{Q}$ with $\left|E\left(K_{\infty}\right)\right|=|E(\mathbb{Q})|=1$; such a $K_{\infty}$ is a big field that is not large. This concludes the proof of Theorem 1.3 .

\section{Additional REMARKS}

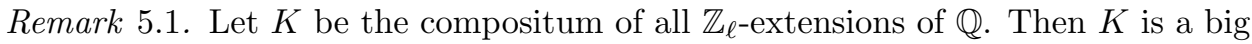
field, and we conjecture that $K$ is not large.

More precisely, let $E$ be an elliptic curve over $\mathbb{Q}$. In [7, Conjecture 10.2] we conjecture that $E(K)$ is finitely generated. Thus combining [7, Conjecture 10.2] with the following theorem of Fehm and Petersen leads to the conjecture that $K$ is not large.

Theorem 5.2 (Fehm and Petersen, Theorem 1.2 of [8]). If $K \subset \overline{\mathbb{Q}}$ is a large field and $A$ is an abelian variety over $K$, then $A(K)$ has infinite rank.

Theorem 5.2 was first proved by Tamagawa in the case that $A$ is an elliptic curve (see the remark at the bottom of page 580 of [8]).

Remark 5.3. By Main Theorem A of [12, Hilbert's Tenth Problem has a negative answer for the ring of integers in any subfield $K$ of $\overline{\mathbb{Q}}$ satisfying

- $K$ is totally real, and

- there is an elliptic curve $E$ over $K$ such that $E(K)$ is finitely generated and has positive rank.

By Theorem 5.2, such a $K$ is not a large field.

Applying Theorem 4.7 with the elliptic curve $E=37 . a 1$ as in Remark 4.3, one can find uncountably many $\mathcal{L}$-towers $K_{\infty} / \mathbb{Q}$ with $K_{\infty}$ totally real and big such that $E\left(K_{\infty}\right)=E(\mathbb{Q}) \cong \mathbb{Z}$. This gives uncountably many non-large big fields over whose ring of integers Hilbert's Tenth Problem has a negative answer.

It is natural to ask whether there is any non-large field over whose ring of integers Hilbert's Tenth Problem has a positive answer.

\section{ACKNOWLEDGMENTS}

Theorem 1.3 answers a question that was brought to our attention by Arno Fehm at the American Institute of Mathematics workshop "Definability and decidability problems in number theory", in May 2019. We are grateful to him for that and for additional helpful correspondence. We also thank the referee for very helpful comments.

\section{REFERENCES}

[1] Zev Klagsbrun, Barry Mazur, and Karl Rubin, Disparity in Selmer ranks of quadratic twists of elliptic curves, Ann. of Math. (2) 178 (2013), no. 1, 287-320, DOI 10.4007/annals.2013.178.1.5. MR3043582

[2] Zev Klagsbrun, Barry Mazur, and Karl Rubin, A Markov model for Selmer ranks in families of twists, Compos. Math. 150 (2014), no. 7, 1077-1106, DOI 10.1112/S0010437X13007896. MR.3230846

[3] Barry Mazur and Karl Rubin, Kolyvagin systems, Mem. Amer. Math. Soc. 168 (2004), no. 799, viii+96, DOI 10.1090/memo/0799. MR.2031496

[4] Barry Mazur and Karl Rubin, Finding large Selmer rank via an arithmetic theory of local constants, Ann. of Math. (2) 166 (2007), no. 2, 579-612, DOI 10.4007/annals.2007.166.579. MR2373150 
[5] B. Mazur and K. Rubin, Ranks of twists of elliptic curves and Hilbert's tenth problem, Invent. Math. 181 (2010), no. 3, 541-575, DOI 10.1007/s00222-010-0252-0. MR2660452

[6] Barry Mazur and Karl Rubin, Diophantine stability, Amer. J. Math. 140 (2018), no. 3, 571616, DOI 10.1353/ajm.2018.0014. With an appendix by Michael Larsen. MR3805014

[7] B. Mazur and K. Rubin, Arithmetic conjectures suggested by the statistical behavior of modular symbols, Exp. Math., to appear, preprint available at arXiv:1910.12798

[8] Arno Fehm and Sebastian Petersen, On the rank of abelian varieties over ample fields, Int. J. Number Theory 6 (2010), no. 3, 579-586, DOI 10.1142/S1793042110003071. MR2652895

[9] The LMFDB Collaboration, The L-functions and Modular Forms Database, http://www. lmfdb.org

[10] Florian Pop, Embedding problems over large fields, Ann. of Math. (2) 144 (1996), no. 1, 1-34, DOI $10.2307 / 2118581$. MR1405941

[11] Jean-Pierre Serre, Propriétés galoisiennes des points d'ordre fini des courbes elliptiques (French), Invent. Math. 15 (1972), no. 4, 259-331, DOI 10.1007/BF01405086. MR387283

[12] Alexandra Shlapentokh, Rings of algebraic numbers in infinite extensions of $\mathbb{Q}$ and elliptic curves retaining their rank, Arch. Math. Logic 48 (2009), no. 1, 77-114, DOI 10.1007/s00153008-0118-y. MR2480937

[13] Joseph H. Silverman, The arithmetic of elliptic curves, Graduate Texts in Mathematics, vol. 106, Springer-Verlag, New York, 1986. MR817210

Department of Mathematics, Harvard University, Cambridge, Massachusetts 02138

Email address: mazur@g.harvard.edu

Department of Mathematics, UC Irvine, Irvine, California 92697

Email address: krubin@uci.edu 